\title{
sciendo
}

\section{Independent verification of treatment planning system calculations}

\begin{abstract}
Purpose: According to the available international recommendations, at least one independent verification of the calculations of number of monitor unit (MU) is required for every patient treated by teleradiotherapy. The aim of this study was to estimate the differences of dose distributions calculated with two treatment planning systems: Eclipse (Varian) and Oncentra MasterPlan (Elekta). Materials and methods: The analysis was performed for 280 three-dimensional conformal radiotherapy treatment (3D-CRT) plans with photon beams from Varian accelerators: CL 600C/D X6 MV (109 plans), CL 2300C/D X6 MV (43 plans), and CL 2300C/D X15 MV (128 plans). The mean doses in the planning target volume (PTV) and doses at the isocenter point obtained with Eclipse and Oncentra MasterPlan (OMP) were compared with Wilcoxon matched-pairs signed rank test. Additionally, the treatment planning system (TPS) calculations were compared with dosimetric measurements performed in the inhomogeneous phantom. Results: Data were analysed for 6 MV plans and for 15 MV plans separately, independently of the treatment machine. The dose values calculated in Eclipse were significantly $(p<0.001)$ higher compared to calculations of OMP system. The average difference of the mean dose to PTV was $(1.4 \pm 1.0) \%$ for X6 MV and $(2.5 \pm 0.6) \%$ for X15 MV. Average dose disparities at the isocenter point were $(1.3 \pm 1.9) \%$ and $(2.1 \pm 1.0) \%$ for X6 MV and X15 MV beams, respectively. The largest differences were observed in lungs, air cavities, and bone structures. Moreover the variation in dosimetric measurements was less as compared to Eclipse calculations. Conclusions: OMP calculations were introduced as the independent MU verification tool with the first action level range equal to 3.5\%.
\end{abstract}

Keywords: 3D-conformal radiotherapy $\bullet$ Treatment planning system $\bullet$ Quality control $\bullet$ Validation of dose calculation $\bullet$ Pre-treatment verification

\section{E. Dąbrowska-Szewczyk ${ }^{凶}$}

Maria Sklodowska-Curie Memorial Cancer

Center and Institute of Oncology

Department of Medical Physics

W. K. Roentgena 5 Str., 02-781 Warsaw, Poland and University of Warsaw

Faculty of Physics, Biomedical Physics Division Pasteura 5 Str., 02-093 Warsaw, Poland

E-mail: e.dabrowska@zfm.coi.pl

A. Zawadzka, A. Walewska, P. Kukołowicz Maria Sklodowska-Curie Memorial Cancer Center and Institute of Oncology

Department of Medical Physics

W. K. Roentgena 5 Str., 02-781 Warsaw, Poland

B. Brzozowska

University of Warsaw

Faculty of Physics, Biomedical Physics Division Pasteura 5 Str., 02-093 Warsaw, Poland

Received: 10 April 2019

Accepted: 2 November 2020

\section{Introduction}

The aim of radiotherapy is to deliver the prescribed dose to the target volume according to the treatment plan. So, effective and safe radiotherapy should be as accurate as possible in every stage of the treatment preparation and delivery process. The calculation of the number of MUs is an important step in the chain of activities leading to the final stage of computerized treatment plan preparation. According to the international recommendations, at least one independent verification of the calculation of the number of MUs is required for each and every patient treated with external radiotherapy. There are several guidelines for MU calculation verification [1]. The simplest and the fastest method of MU verification is by using a hand-held simple PC software [2]. In Poland, the quality assurance (QA) of radiotherapy is regulated by the Regulation of the Polish Minister of Health [3]. The simplest method, a simple hand-held PC software, has usually several disadvantages, such as not taking into account patient geometry, tissue heterogeneities, the field-shaping device

0029-5922 @ 2021 The Author(s). Published by the Institute of Nuclear Chemistry and Technology.

This is an open access article under the CC BY-NC-ND 4.0 licence (http://creativecommons.org/licences/by-nc-nd/4.0/). 
(MLC, blocks aperture), etc. More advanced solutions available commercially are independent of the MU verification calculation software, such as RadCalc [4], DIAMOND [5] or Mobius3D [6], where patient geometry and beam shape are considered. The dose delivered to a patient can also be verified by different methods of in vivo dosimetry [7]. However, the most advanced method of dose calculation verification is calculating the dose distribution using a second independent treatment planning system (TPS) as proposed by Report of AAPM Task Group 114 [8]. In our center, two TPSs are available, Eclipse (version 10.0, Varian) and Oncentra MasterPlan (version 3.3, Elekta). In our procedure, the 3D conformal treatment plans were prepared with the Eclipse system and verified with the OMP.

The main goal of this study is to present the comparison results of dose distribution performed with Eclipse and OMP. The doses calculated for PTV with these two systems are presented. We have also presented the results of dosimetric verification of dose distribution calculations carried out with both systems in the inhomogeneous phantom. The problems in the interpretation of results were also discussed.

\section{Materials and methods}

\section{Patient data}

The analysis and assessment of differences in dose calculations obtained with two treatment planning systems (TPSs) were performed for patients treated with $6 \mathrm{MV}$ and $15 \mathrm{MV}$ X-rays, respectively, in our radiotherapy center. The results of calculations for randomly selected patients were analysed in this study. Patients were irradiated at the following treatment sites: head and neck, brain, breast, oesophagus, lung, gynecology, prostate, rectum, soft tissues (sarcomas), and others. Further, a three-dimensional conformal radiotherapy (3D-CRT) plan was prepared for each patient. All treatment plans were delivered on a Varian CL $600 \mathrm{C} / \mathrm{D}$ (X6 MV) or CL $2300 \mathrm{C} / \mathrm{D}$ (X6 MV/X15 MV) accelerators. The differences were analysed for $6 \mathrm{MV}$ and $15 \mathrm{MV}$ energies separately. There were 152 plans for $6 \mathrm{MV}$ beams and 128 plans for $15 \mathrm{MV}$ beams (see Table 1).

Computed tomography (CT) was carried out for each patient according to the internal protocols used in our hospital. Subsequently, GTV/CTV/ PTV (gross tumour volume/clinical target volume/ planning target volume) and organs at risk (OARs) were delineated on $3 \mathrm{~mm}$-thick transversal slices for patients with brain or head and neck cancer and $5 \mathrm{~mm}$-thick transversal slices in case of other localizations. All structures were delineated according to

Table 1. The number of analysed plans according to calculation models

\begin{tabular}{|c|c|}
\hline Energy & Number of treatment plans \\
\hline$\overline{\mathrm{X} 6 \mathrm{MV}}$ & 152 \\
\hline X15 MV & 128 \\
\hline
\end{tabular}

ICRU recommendation $[9,10]$. The following cases were analysed:

a) head and neck: two perpendicular or oblique wedged fields,

b) brain: non-standard geometry; two to four beams,

c) breast: two opposed wedged beams with the medial field border aligned to avoid irradiation of heart and ipsilateral lung,

d) oesophagus: three wedged fields,

e) lung: three to five fields, some of them with wedges,

f) gynecology: box technique or three fields (open anteroposterior (AP) and two lateral wedged fields),

g) prostate, rectum: three wedged fields (open AP and two lateral wedged fields), and

h) soft tissues: the non-standard geometry of beams. All treatment beams were set up isocentrically.

\section{Dose calculation algorithms and TPSs}

For each patient, a 3D-CRT treatment plan was prepared in the Eclipse TPS. The analytical anisotropic algorithm (AAA) [11] with $2.5 \mathrm{~mm}$ grid size was used for the calculation of dose distribution. Each treatment plan was prepared according to ICRU Reports no. 50 and 62 and the internal protocols were used for OARs. Further, the dose distribution in primary TPS (Eclipse) was always normalized to the mean dose in the PTV. Subsequently, the DICOM RT data were transferred to OMP, and the dose distribution was calculated. In the OMP, the collapse cone convolution (CCC) algorithm [12] with $2 \mathrm{~mm}$ cubic grid was used for the second dose distribution calculation. Also, the same number of MUs was utilized for each beam.

\section{Commissioning of Eclipse and OMP}

Both TPSs were carefully checked prior to the first clinical use. The input data used for modelling of Varian accelerators beams, the percent depth doses, profiles and output factors were calculated using water phantom for both TPSs and compared with the input data. The gamma method with gamma $2 \mathrm{~mm}, 2 \%$ was used to compare percent depth doses and profiles. The gamma values larger than 1 were obtained in the build-up region and for the smallest beam sizes, i.e. $2 \mathrm{~cm} \times 2 \mathrm{~cm}$ and $3 \mathrm{~cm} \times 3 \mathrm{~cm}$ only. The actual CT conversion HU-electron density curve was entered into both systems.

\section{Data analysis}

Data were analysed for $6 \mathrm{MV}$ plans and for $15 \mathrm{MV}$ plans separately. For each plan, the dose disparity between Eclipse and OMP calculations were expressed in terms of the difference between mean doses in the PTV and the doses at the isocenter point. The dose difference was calculated using the formula (1): 


$$
\Delta_{k}=\left(\frac{x^{\mathrm{E}}}{x^{\mathrm{OMP}}}-1\right) \cdot 100 \%
$$

where: $k$ - plan's number, $x^{\mathrm{E}}, x^{\mathrm{OMP}}-$ dose statistics computed with Eclipse and OMP for plan number $k$, respectively.

Further, the mean of differences (2) and the standard deviation (STD) of these differences (3) were calculated, for each group of plans, using the following formulas:

$$
\begin{gathered}
\bar{\Delta}=\frac{1}{k} \sum_{n=1}^{k} \Delta_{k} \\
S_{\bar{\Delta}}=\sqrt{\frac{1}{(k-1) \cdot k} \sum_{n=1}^{k}\left(\bar{\Delta}-\Delta_{k}\right)^{2}}
\end{gathered}
$$

Note that the Wilcoxon matched-pairs signed rank test was used for statistical analyses of differences. The differences were considered as statistically significant if $p<0.001$ (two-tailed). All statistical analyses were performed using SPSS software (version 19.0, IBM Corporation).

\section{Dose calculation with and without inhomogeneity correction}

Additional calculations without inhomogeneity correction were performed for 24 plans in which the dose distribution was highly influenced by inhomogeneities; further, large dose differences between Eclipse and OMP calculations were obtained. The disparity of the mean dose in the PTV and dose at the isocenter point were computed using formulas (1), (2), and (3).

\section{Dosimetric measurements}

The calculations of both TPSs were compared with measurements which were performed using the inhomogeneous phantom [13]. The phantom is presented in Fig. 1. The phantom was the solid polystyrene phantom of $15 \mathrm{~cm}$ width, $15 \mathrm{~cm}$ length and $15 \mathrm{~cm}$ height. The physical density was $1.04 \mathrm{~g} / \mathrm{cm}^{3}$, and the relative electron density to water was 1.00 . One of the polystyrene slabs allows the positioning

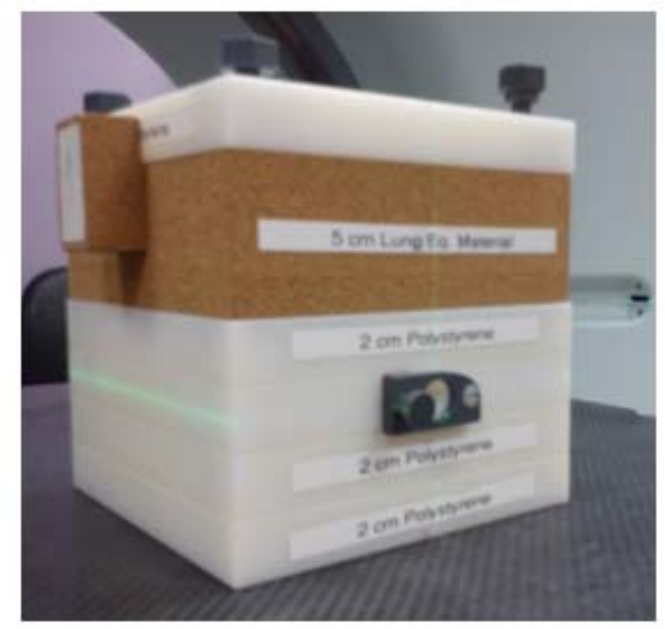

Fig. 1. Polystyrene/lung solid phantom. of the ionization chamber in the phantom. Phantom also consists of two exchangeable inhomogeneity slabs to mimic the bone $(15 \mathrm{~cm} \times 15 \mathrm{~cm} \times 2 \mathrm{~cm})$ and lung $(15 \mathrm{~cm} \times 15 \mathrm{~cm} \times 5 \mathrm{~cm})$. The measurements were performed with the calibrated cylindrical ionization chamber TM 31010 (PTW) with a sensitive volume of $0.125 \mathrm{~cm}^{3}$, connected to Unidos (PTW) electrometer. An ionization chamber was placed at a physical depth of $10 \mathrm{~cm}$.

Three phantom configurations (polystyrene, polystyrene with lung-equivalent material, and polystyrene with bone-equivalent material) were CT scanned. All acquired images were exported to Eclipse TPS. During CT imaging, the ionization chamber slot was filled with solid water. Also, for each phantom configuration, six various geometries of treatment plans were prepared (Table 2) for both $6 \mathrm{MV}$ and $15 \mathrm{MV}$ photon beams. DICOM RT data for each plan was transferred to OMP TPS where dose distribution was calculated.

After the dose calculations in Eclipse and OMP TPSs, irradiations of the solid phantom in three configurations were performed with Varian CL 2300 $\mathrm{C} / \mathrm{D}$. The numbers of MUs were calculated to deliver $2 \mathrm{~Gy}$ at the isocenter point. The actual output factor was taken into account.

The measured doses were compared with the doses calculated in Eclipse and OMP for each of the following, namely, the phantom configuration, energy, and geometry of the treatment plan. The comparison was performed using the equation:

$$
\Delta_{\text {MEASUREMENT }}^{\mathrm{TPS}}=\left(\frac{x^{\mathrm{TPS}}}{x^{\text {MEASUREMENT }}}-1\right) \cdot 100 \%
$$

where: $x^{\mathrm{TPS}}$ - dose computed by treatment planning system, $x^{\text {MEASUREMENT }}-$ measured dose.

\section{Results}

Tables 3 and 4 present the differences between the mean dose to the PTV and dose to isocenter calculated with Eclipse and OMP for $6 \mathrm{MV}$ and $15 \mathrm{MV}$ beams. The results are given separately in terms of the mean value \pm standard deviations of differences for each type of energy. The distributions of these differences are presented in Figs. 2 and 3.

Table 5 presents the differences between mean doses and doses to isocenter calculated in both systems for 24 plans in which inhomogeneities highly influenced on dose distribution. The mean doses and the doses to isocenter were calculated for both with and without inhomogeneity correction. If inhomogeneity correction was applied, the mean values of differences exceeded $1 \%$ and $2 \%$ for $6 \mathrm{MV}$ and 15 MV beams, respectively. However, if inhomogeneity correction was not applied for $6 \mathrm{MV}$ beams, the mean value differences between the mean doses to the PTV, and doses to isocenter, was smaller than $0.5 \%$. Also, for $15 \mathrm{MV}$ beams, these differences were also smaller with inhomogeneity correction.

Table 6 presents the comparison of doses measured using the phantom and calculated in both TPSs. For Eclipse system, the differences measured 
Table 2. Geometries of treatment plans used for measurements

\begin{tabular}{|c|c|c|c|c|c|}
\hline No. & $\begin{array}{l}\text { Number } \\
\text { of fields }\end{array}$ & $\begin{array}{c}\text { Gantry } \\
\text { directions }\end{array}$ & $\begin{array}{l}\text { Size of field } \\
(\mathrm{cm} \times \mathrm{cm})\end{array}$ & Wedge & $\begin{array}{l}\text { Shape } \\
\text { of field }\end{array}$ \\
\hline & \multicolumn{5}{|c|}{ Polystyrene } \\
\hline 1 & 1 & $0^{\circ}$ & $3 \times 3$ & None & square \\
\hline 2 & 1 & $0^{\circ}$ & $8 \times 8$ & None & square \\
\hline 3 & 3 & $0^{\circ}, 90^{\circ}, 270^{\circ}$ & $8 \times 8,8 \times 8,8 \times 8$ & None & square \\
\hline 4 & 3 & $0^{\circ}, 70^{\circ}, 290^{\circ}$ & $8 \times 8,8 \times 8,8 \times 8$ & None & circular \\
\hline 5 & 3 & $0^{\circ}, 70^{\circ}, 290^{\circ}$ & $8 \times 8,8 \times 8,8 \times 8$ & none, $45 \mathrm{~L}, 45 \mathrm{R}$ & circular \\
\hline 6 & 3 & $0^{\circ}, 70^{\circ}, 290^{\circ}$ & $8 \times 8,8 \times 8,8 \times 8$ & none, 45 OUT, 45 IN & circular \\
\hline \multicolumn{6}{|c|}{ Polystyrene with bone equivalent material } \\
\hline 7 & 1 & $0^{\circ}$ & $3 \times 3$ & None & square \\
\hline 8 & 1 & $0^{\circ}$ & $8 \times 8$ & None & square \\
\hline 9 & 3 & $0^{\circ}, 90^{\circ}, 270^{\circ}$ & $8 \times 8,10 \times 8,10 \times 8$ & None & square \\
\hline 10 & 3 & $0^{\circ}, 45^{\circ}, 315^{\circ}$ & $8 \times 8,9.5 \times 8,9.5 \times 8$ & None & circular \\
\hline 11 & 3 & $0^{\circ}, 45^{\circ}, 315^{\circ}$ & $8 \times 8,9 \times 8,9 \times 8$ & none, $45 \mathrm{~L}, 45 \mathrm{R}$ & circular \\
\hline 12 & 3 & $0^{\circ}, 45^{\circ}, 315^{\circ}$ & $8 \times 8,8 \times 9,8 \times 9$ & none, 45 OUT, 45 IN & circular \\
\hline \multicolumn{6}{|c|}{ Polystyrene with lung equivalent material } \\
\hline 13 & 1 & $0^{\circ}$ & $3 \times 3$ & None & square \\
\hline 14 & 1 & $0^{\circ}$ & $8 \times 8$ & None & square \\
\hline 15 & 3 & $0^{\circ}, 90^{\circ}, 270^{\circ}$ & $8 \times 8,9.5 \times 8,9.5 \times 8$ & None & square \\
\hline 16 & 3 & $0^{\circ}, 50^{\circ}, 310^{\circ}$ & $8 \times 8,9 \times 8,9 \times 8$ & None & circular \\
\hline 17 & 3 & $0^{\circ}, 60^{\circ}, 300^{\circ}$ & $8 \times 8,9 \times 8,9 \times 8$ & none, $45 \mathrm{~L}, 45 \mathrm{R}$ & circular \\
\hline 18 & 3 & $0^{\circ}, 50^{\circ}, 310^{\circ}$ & $8 \times 8,8 \times 9,8 \times 9$ & none, 45 OUT, 45 IN & circular \\
\hline
\end{tabular}

with homogenous polystyrene phantom and with polystyrene phantom under lung and bone inhomogeneity are found to be $2 \%$. The largest differences were obtained for bone. Much smaller differences were obtained for the OMP system. For the OMP, the discrepancies between measurements and calculations were smaller than $1 \%$, except in three cases for X6 MV and three cases for X15 MV.

Table 3. Differences between Eclipse and OMP calculations of mean doses to the PTV

\begin{tabular}{lcccc}
\hline \multicolumn{1}{r}{ Energy } & Cases & Average (\%) \pm STD (\%) & Range (\%) & $p$-value $(p<0.001)$ \\
\hline X6 MV & 152 & $1.4 \pm 1.0$ & $-3.4,6.0$ & 0.0 \\
X15 MV & 128 & $2.5 \pm 0.6$ & $-0.2,4.5$ & 0.0 \\
\hline
\end{tabular}

Table 4. Differences between Eclipse and OMP calculations of dose to isocenter

\begin{tabular}{lcccc}
\hline \multicolumn{1}{r}{ Energy } & Cases & Average $(\%) \pm$ STD $(\%)$ & Range (\%) & $p$-value $(p<0.001)$ \\
\hline X6 MV & 152 & $1.3 \pm 1.9$ & $-2.7,10.6$ & 0.0 \\
X15 MV & 128 & $2.1 \pm 1.0$ & $-0.7,5.6$ & 0.0 \\
\hline
\end{tabular}

Table 5. Statistical summary of differences between Eclipse and OMP calculation with and without inhomogeneity correction

\begin{tabular}{|c|c|c|c|c|}
\hline Energy & Plans & Average $(\%) \pm$ STD $(\%)$ & Range (\%) & $p$-value $(p<0.001)$ \\
\hline \multicolumn{5}{|c|}{ Mean dose with inhomogeneity correction } \\
\hline X6 MV & 24 & $1.3 \pm 0.5$ & $0.1,2.1$ & 0.000 \\
\hline $\mathrm{X} 15 \mathrm{MV}$ & 24 & $2.6 \pm 0.6$ & $1.6,4.5$ & 0.000 \\
\hline \multicolumn{5}{|c|}{ Dose at isocenter with inhomogeneity correction } \\
\hline X6 MV & 24 & $1.1 \pm 1.5$ & $-1.0,6.8$ & 0.000 \\
\hline X15 MV & 24 & $2.2 \pm 1.1$ & $0.4,5.2$ & 0.000 \\
\hline \multicolumn{5}{|c|}{ Mean dose without inhomogeneity correction } \\
\hline X6 MV & 24 & $0.4 \pm 0.4$ & $-0.8,1.0$ & 0.000 \\
\hline X15 MV & 24 & $1.0 \pm 0.3$ & $0.5,1.6$ & 0.000 \\
\hline \multicolumn{5}{|c|}{ Dose in isocenter without inhomogeneity correction } \\
\hline X6 MV & 24 & $0.2 \pm 0.7$ & $-1.0,2.4$ & 0.110 \\
\hline X15 MV & 24 & $0.5 \pm 0.9$ & $-2.1,2.8$ & 0.001 \\
\hline
\end{tabular}




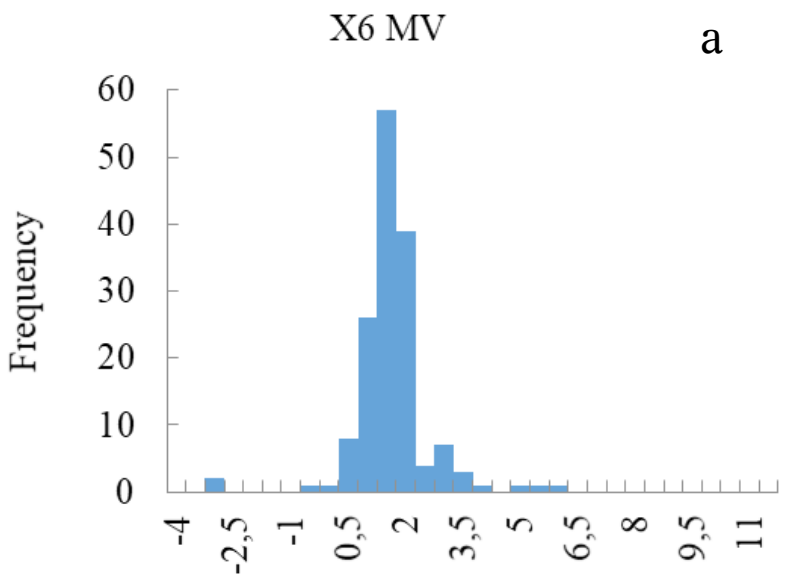

X15 MV

b

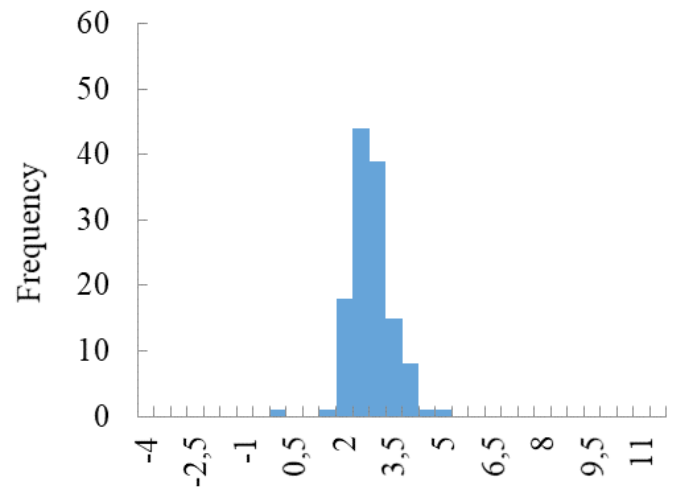

Fig. 2. Frequency histograms of differences between Eclipse and OMP calculations of mean doses to the PTV for 6 MV (a) and $15 \mathrm{MV}(\mathrm{b})$.
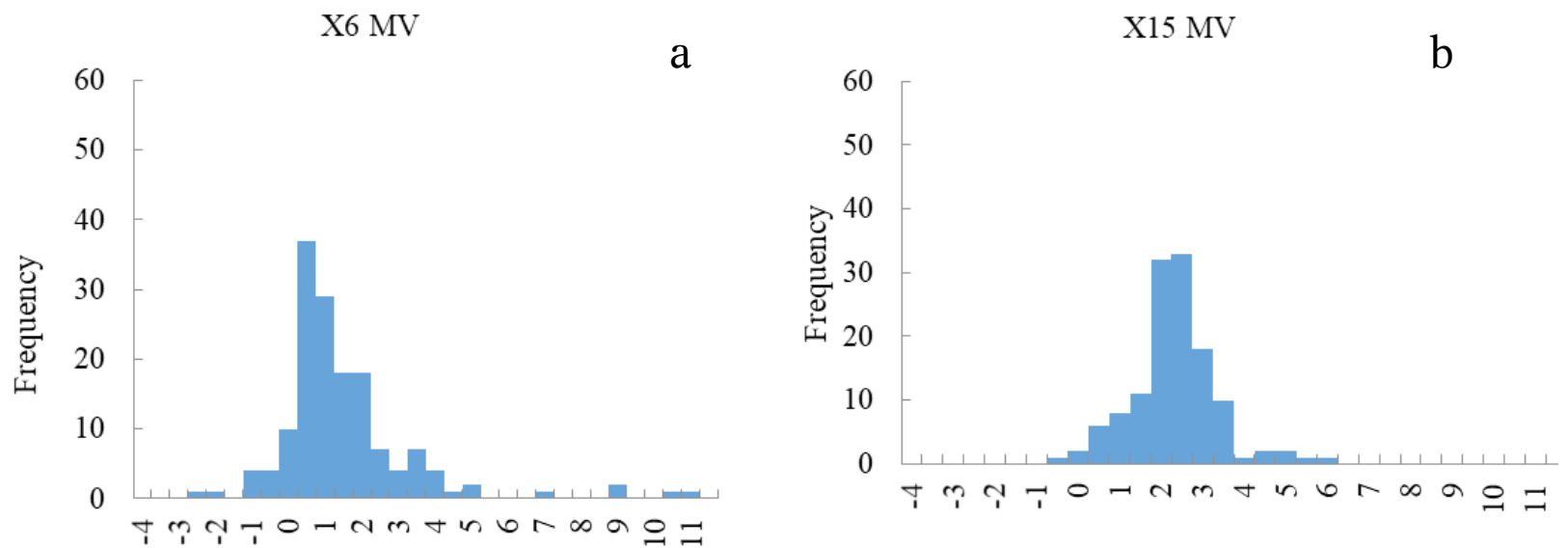

Fig. 3. Frequency histograms of differences between Eclipse and OMP calculations of doses to isocenter for 6 MV (a) and $15 \mathrm{MV}$ beams (b).

Table 6. The discrepancy between Eclipse and OMP calculations and measurements for various beams geometry

\begin{tabular}{|c|c|c|c|c|}
\hline \multirow{2}{*}{ No. } & \multicolumn{2}{|c|}{ X6 MV } & \multicolumn{2}{|c|}{ X15 MV } \\
\hline & $\Delta_{\text {MEASUREMENT }}^{\text {ECLIPSE }}(\%)$ & $\Delta_{\text {MEASUREMENT }}^{\text {OMP }}(\%)$ & $\Delta_{\text {MEASUREMENT }}^{\text {ECLIPSE }}(\%)$ & $\Delta_{\text {MEASUREMENT }}^{\text {OMP }}(\%)$ \\
\hline \multicolumn{5}{|c|}{ Polystyrene phantom } \\
\hline 1 & 2.2 & 0.3 & 2.2 & 0.8 \\
\hline 2 & 2.2 & -0.5 & 2.8 & -0.4 \\
\hline 3 & 2.0 & 0.2 & 3.0 & 0.4 \\
\hline 4 & 2.4 & 0.4 & 2.8 & 0.1 \\
\hline 5 & 2.1 & -0.1 & 2.1 & -0.3 \\
\hline 6 & 2.1 & -0.1 & 2.3 & -0.4 \\
\hline \multicolumn{5}{|c|}{ Polystyrene with bone equivalent material } \\
\hline 7 & 2.5 & 1.4 & 2.5 & 1.4 \\
\hline 8 & 2.3 & 0.5 & 3.6 & 0.8 \\
\hline 9 & 2.6 & 0.6 & 2.6 & -0.1 \\
\hline 10 & 2.8 & 0.8 & 4.0 & 1.3 \\
\hline 11 & 2.8 & 0.8 & 3.5 & 1.0 \\
\hline 12 & 2.4 & 0.6 & 3.4 & 0.8 \\
\hline \multicolumn{5}{|c|}{ Polystyrene with lung equivalent material } \\
\hline 13 & 2.9 & 1.3 & 1.5 & 0.7 \\
\hline 14 & 3.3 & -0.3 & 3.1 & -0.4 \\
\hline 15 & 1.7 & -0.2 & 2.3 & -0.2 \\
\hline 16 & 2.9 & 1.4 & 2.9 & 0.8 \\
\hline 17 & 1.8 & 0.6 & 2.2 & 0.3 \\
\hline 18 & 1.6 & 0.2 & 2.3 & 0.2 \\
\hline
\end{tabular}




\section{Discussion}

The independent verification of the MU calculation for 3D-CRT plan is recommended or even required by law. In our center, verification is performed by comparing the dose distribution calculations carried out with Eclipse and with OMP. Further, the comparison results are presented for $6 \mathrm{MV}$ and $15 \mathrm{MV}$ beams, separately. Also, the mean doses to the PTV and the doses to isocenter are compared.

On the average, the mean doses to the PTV calculated with Eclipse were larger than those calculated with OMP of $1.4 \%$ and $2.5 \%$ for $6 \mathrm{MV}$ and $15 \mathrm{MV}$ beams, respectively. Similar results were obtained for the doses to isocenter. A very similar result was obtained for the differences of dose to isocenter (Fig. 3), but the dispersion of differences is larger for the dose to isocenter than for the mean dose i.e., $1.9 \%$ vs. $1.0 \%$ for $6 \mathrm{MV}$ and $1.0 \%$ vs. $0.6 \%$ for $15 \mathrm{MV}$. All the differences were statistically significant. The variations obtained between both systems were smaller than 3.5\% for almost all plans in the mean doses for $6 \mathrm{MV}$. For $15 \mathrm{MV}$ beams, these discrepancies were larger than $3.5 \%$ in 30 out of 128 plans. As for the doses to isocenter, for $6 \mathrm{MV}$ and $15 \mathrm{MV}$ beams, those discrepancies which amounted to larger than $3.5 \%$ were observed for 25 plans and 11 plans, respectively. Before getting the results published in this work, the $3.5 \%$ discrepancy has been treated as the first action level. According to our rules, when the discrepancy amounts to larger than $3.5 \%$, the case should be analysed carefully. Next, since this large discrepancy could not be explained, the procedure used for intensity modulated radiation therapy (IMRT) plans was followed. The verification plan was prepared and measured with Octavius dosimetry system (PTW). The results of measurements did not all exceed the action level.

After getting the results obtained in this work, the first action level was changed to the range of $(-1.5 \%, 5.5 \%)$. Our decision is based on the comparison of calculations and measurements in the inhomogeneous phantom. The calculation doses performed with Eclipse were systematically larger than the measurements of about 2-3\% for both energies, while for OMP, these differences were much smaller. These results were in a sense consistent with the differences of calculations obtained for patients with both systems. The dose calculated in the OMP was almost always smaller on the average of 1.5\% than in Eclipse. Also, we want to stress that, for both systems, comparisons of calculations and measurements carried out in a water phantom for many different sizes of beams and geometries provided an excellent result. The conclusion has been drawn that for Eclipse, the discrepancy between calculations and measurements has been the result of inhomogeneity correction. This conclusion is substantiated by using the results of the comparison obtained for 24 plans for which we performed the comparison again with and without inhomogeneity correction. When the inhomogeneity correction is switched off, the discrepancy between both systems is decreased by about $1 \%$ on an average. There might be at least two reasons for these discrepancies which were obtained for inhomogeneity. The first source of wrong calculations might be the erroneous HU-electron density curve used for performing calculations. The HU-electron density curve was measured, and we did not find any differences between the curve entered into the systems and the measured curve. The second reason might be the unique algorithms implemented for inhomogeneity calculations in the TPSs. The user has no influence on the algorithm used in the system.

According to recommendations, calculations should be checked for the number of MUs. The easiest way is to calculate the dose distribution or the number of MUs with another software. It is worth noting that this procedure may be unreliable and may lead to wrong decisions. Both programs may calculate the dose distribution with an error. Both systems may deliver the number of MUs as being larger or smaller than the correct ones. Subsequently, the user may receive the false positive results when comparing the results obtained with both systems. If one system calculates a larger number of MUs while the second system calculates a smaller number, the user may obtain a false negative result. Therefore, prior to using any program, it should always be validated. The validation should be carried out by the comparison of measurements and calculations. It is relatively easy to make measurements in the homogeneous phantom. It is much more difficult to perform measurements in the inhomogeneous phantom. Nevertheless, such an effort should always be made. The results of our measurements have revealed that the calculations carried out in the OMP are slightly more trustworthy. Consequently, we have decided to change the action level region.

\section{Conclusions}

The MU calculations have been verified with two different 3D TPSs, but the calculations carried out with these systems always require to be validated. The definition of the action level should account for the results of verification of the accuracy of calculation of each TPS. A verification procedure should also be performed in the inhomogeneous phantom.

Funding. This work was partially supported by the Polish Funds for Science.

Conflict of interest. All authors declare that they have no conflict of interest.

\section{ORCID}

B. Brzozowska (10 http://orcid.org/0000-0003-0247-697X

E. Dabrowska-Szewczyk (1) http://orcid.org/0000-00024193-8471

P. Kukołowicz (10 http://orcid.org/0000-0001-9050-0161

A. Walewska (1) http://orcid.org/0000-0002-3645-3736

A. Zawadzka (D) http://orcid.org/0000-0003-0757-0869 


\section{References}

1. European Union. (2013). Council Directive 2013/59/ Euratom of 5 December 2013 laying down basic safety standards for protection against the dangers arising from exposure to ionising radiation, and repealing Directives 89/618/Euratom, 90/641/Euratom, 96/29/ Euratom, 97/43/Euratom and 2003/122/Euratom. Official Journal of the European Union, OJ L13, 17.1.2014, 1-73. https://eur-lex.europa.eu/legalcontent/EN/TXT/?uri=OJ:L:2014:013:TOC.

2. Knöös, T., Johnsson, S. A., Ceberg, C. P., Tomaszewicz, A., \& Nilsson, P. (2001). Independent checking of the delivered dose for high-energy X-rays using a hand-held PC. Radiother. Oncol., 58(2), 201-208.

3. Polish Minister of Health. (2011). Regulation of the Minister of Health of 18 February 2011 on the conditions for safe use of ionizing radiation for all types of medical exposure. Dz. U., 2011, no. 51, item 265. (in Polish).

4. Haslam, J. J., Bonta, D. V., Lujan, A. E., Rash, C., Jackson, W., \& Roeske, J. C. (2003). Comparison of dose calculated by an intensity modulated radiotherapy treatment planning system and an independent monitor unit verification program. J. Appl. Clin. Med. Phys., 4(3), 224-230.

5. Mata Colodro, F., Serna Berná, A., \& Puchades Puchades, V. (2013). Dosimetric validation of a redundant independent calculation software for VMAT fields. Phys. Med., 29(4), 341-349.

6. Fontenot, J. D. (2014). Evaluation of a novel secondary check tool for intensity-modulated radiotherapy treatment planning. J. Appl. Clin. Med. Phys., 15, 207-215.

7. Mijnheer, B., Beddar, S., Izewska, J., \& Reft, C. (2013) In vivo dosimetry in external beam radiotherapy. Med. Phys., 40(7), 070903.

8. Stern, R. L., Heaton, R., Fraser, M. W., Goddu, S. M., Kirby, T. H., Lam, K. L., Molineu, A., \& Zhu, T. C. (2011). Verification of monitor unit calculations for non-MRT clinical radiotherapy: Report of AAPM Task Group 114. Med. Phys., 38(1), 504-530.

9. Landberg, T., Chavaudra, J., Dobbs, J., Hanks, G., Johansson, K. A., Möller, T., \& Purdy, J. (1993). Report 50: Prescribing, recording and reporting photon beam therapy. Journal of the International Commission on Radiation Units and Measurements, 26(1):NP.

10. Landberg, T., Chavaudra, J., Dobbs, J., Gerard, J. P., Hanks, G., Horiot, J. C., Johansson, K. A., Möller, T. Purdy, J., Suntharalingam, N., \& Svensson, H. (1999). Report 62: Prescribing, recording and reporting photon beam therapy. Journal of the International Commission on Radiation Units and Measurements, 32(1):NP.

11. Sievinen, J., Ulmer, W., \& Kaissl, W. (2005). AAA Photon dose calculation model in Eclipse. Varian Medical Systems.

12. Ahnesjö, A. (1989). Collapsed cone convolution of radiant energy for photon dose calculation in heterogeneous media. Med. Phys., 16(4), 577-592.

13. Bulski, W., Chełmiński, K., \& Rostkowska, J. (2015). Dosimetry audit of radiotherapy treatment planning systems. Radiat. Prot. Dosim., 165(1/4), 472-476. 